EVS29 Symposium

Montréal, Québec, Canada, June 19 - 22, 2016

\title{
Dynamic Wireless Power Transfer System for Electric Vehicles to Simplify Ground Facilities - Real-time Power Control and Efficiency Maximization -
}

\author{
Katsuhiro Hata ${ }^{1}$, Daita Kobayashi ${ }^{1}$, Takehiro Imura ${ }^{1}$, Yoichi Hori ${ }^{1}$ \\ ${ }^{1}$ The University of Tokyo, 5-1-5, Kashiwanoha, Kashiwa, Chiba, Japan, hata@hflab.k.u-tokyo.ac.jp
}

\begin{abstract}
This paper focuses on dynamic wireless power transfer for electric vehicles and proposes a vehicle-side control method for real-time power control and efficiency maximization. The proposed control strategy and controller design are presented based on a real-time estimation of the mutual inductance between a transmitter and a receiver. Simulations and experiments demonstrate that the proposed method can achieve the maximum efficiency and the desired power simultaneously.
\end{abstract}

Keywords: wireless charging, dynamic charging, EV (electric vehicle), efficiency, power

\section{Introduction}

Wireless power transfer (WPT) is one of the hottest research topic in transportation applications [1, 2]. In particular, a dynamic wireless power transfer (DWPT) system for electric vehicles (EVs) has gathered attention to extend the cruising distance of EVs and to reduce the size of the energy storage system [3]-[6]. Its ground facilities are mainly composed of power source, high-frequency inverters, transmitters, and so on. As they are applied to rugged roadways over long distances, power control and efficiency maximization of wireless charging are desirable to be achieved on the vehicle side without signal communication.

Although previous research has proposed simultaneous control methods of power control and efficiency maximization on the vehicle side [7,8], they have not been applied to the DWPT system. For maximizing the transmitting efficiency in the DWPT system, the mutual inductance between a transmitter and a receiver has to be estimated from the vehicle side. In this paper, an estimation method considering the vehicle-side control is proposed and applied to the simultaneous power and efficiency control. The effectiveness of the proposed method is verified by simulations and experiments.

\section{Wireless Power Transfer via Magnetic Resonance Coupling}

\subsection{Circuit analysis}

The transmitter and the receiver coils are shown in Figure 1. They are compensated by resonance capacitors for WPT via magnetic resonance coupling [9], which can achieve a highly efficient mid-range transmission and robustness to misalignment. In this paper, a series-series (SS) compensated circuit topology is used and its circuit diagram is shown in Figure 2 [10]. The transmitter and the receiver are characterized by the inductances $L_{1}, L_{2}$, the series-resonance capacitances $C_{1}, C_{2}$, and the internal 


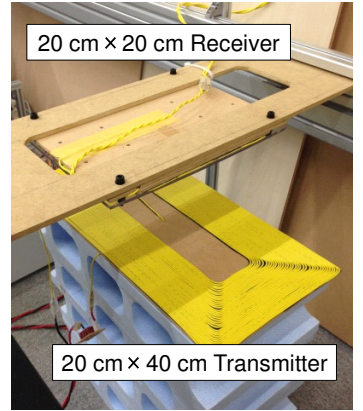

Figure 1: Coils.
Table 1: Parameters of Coils.

\begin{tabular}{l|c|c}
\hline & Transmitter & Receiver \\
\hline Resistance $R_{1}, R_{2}$ & $1.95 \Omega$ & $1.60 \Omega$ \\
Inductance $L_{1}, L_{2}$ & $417.1 \mu \mathrm{H}$ & $210.6 \mu \mathrm{H}$ \\
Capacitance $C_{1}, C_{2}$ & $6030 \mathrm{pF}$ & $12110 \mathrm{pF}$ \\
\hline Resonant frequency $f_{1}, f_{2}$ & $100.4 \mathrm{kHz}$ & $99.7 \mathrm{kHz}$ \\
\hline Coil gap & \multicolumn{2}{|c}{$100 \mathrm{~mm}$} \\
\hline Mutual inductance $L_{m}$ & $36.3 \mu \mathrm{H}$ (no misalignment) \\
\hline Coupling coefficient $k$ & \multicolumn{2}{|c}{0.122 (no misalignment) } \\
\hline
\end{tabular}

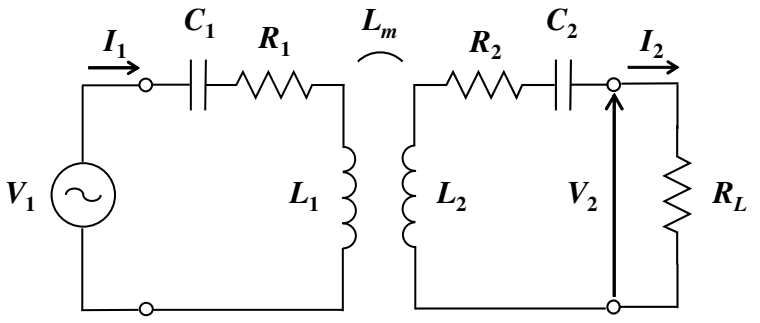

Power source Transmitter and Receiver
Load

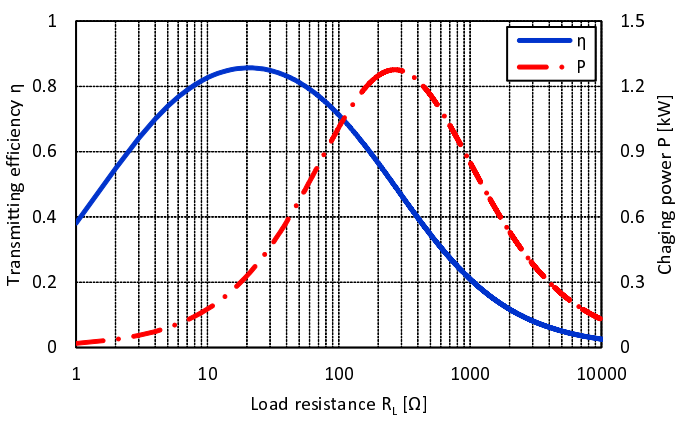

Figure 3: $R_{L}$ vs. $\eta$ and $P$.

Figure 2: Equivalent circuit of WPT system.

resistances $R_{1}, R_{2}$, respectively. $L_{m}$ is the mutual inductance between the transmitter and the receiver. These parameters are expressed in Table 1. If power source angular frequency is designed as follows:

$$
\omega_{0}=\frac{1}{\sqrt{L_{1} C_{1}}}=\frac{1}{\sqrt{L_{2} C_{2}}}
$$

the transmitting efficiency $\eta$ and the transmitting power $P$ can be obtained as follows [11]:

$$
\begin{aligned}
& \eta=\frac{\left(\omega_{0} L_{m}\right)^{2} R_{L}}{\left(R_{2}+R_{L}\right)\left\{R_{1} R_{2}+R_{1} R_{L}+\left(\omega_{0} L_{m}\right)^{2}\right\}} \\
& P=\frac{\left(\omega_{0} L_{m}\right)^{2} R_{L}}{\left\{R_{1} R_{2}+R_{1} R_{L}+\left(\omega_{0} L_{m}\right)^{2}\right\}^{2}} V_{1}^{2}
\end{aligned}
$$

where $V_{1}$ is the RMS value of the primary voltage and $R_{L}$ is the load resistance. When $V_{1}$ equals to 100 $\mathrm{V}, \eta$ and $P$ are expressed in Figure 3 . In order to maximize the transmitting efficiency $\eta, R_{L}$ has to be given as follows [11]:

$$
R_{L \eta \max }=\sqrt{R_{2}\left\{\frac{\left(\omega_{0} L_{m}\right)^{2}}{R_{1}}+R_{2}\right\}} .
$$

Then, the transmitting power $P$ is determined by $R_{L \eta \max }$. As a result, the desired power cannot be achieved only using $R_{L}$ optimization when the transmitting efficiency $\eta$ is maximized.

\subsection{System configuration}

In order to achieve the maximum efficiency and the desired power simultaneously, the vehicle is equipped with two power converters, i.e. Half Active Rectifier (HAR) and the DC-DC converter [7, 8]. The circuit diagram of the DWPT system is shown in Figure 4. The ground facility consists of voltage source $V_{S}$ and an inverter, which generates a square wave voltage with resonance angular frequency $\omega_{0}$. The transmitting power $P$ is rectified by the HAR and the charging power $P_{L}$ is controlled by the DC-DC converter. These control strategies and controller design methods are described below. 


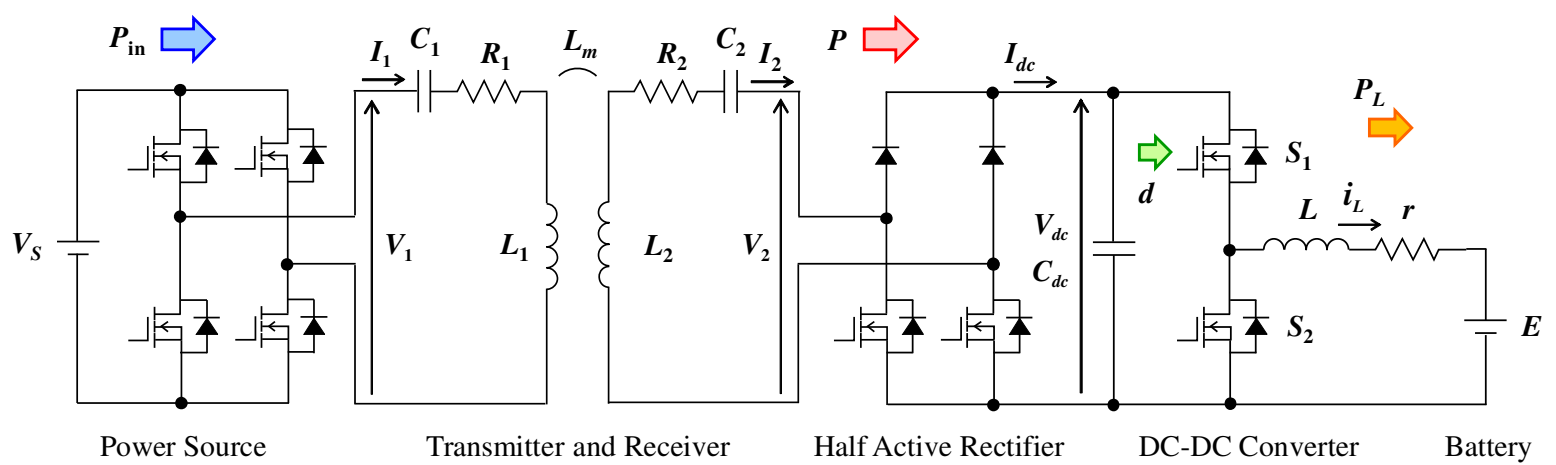

Figure 4: Circuit diagram of the DWPT system.

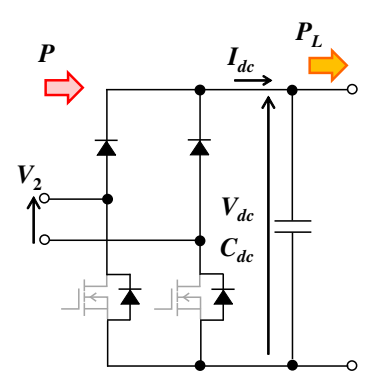

(a) Rectification mode

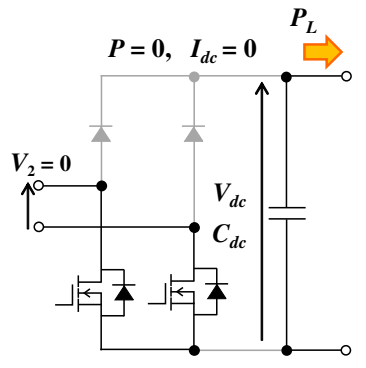

(b) Short mode

Figure 5: Operation modes of Half Active Rectifier.

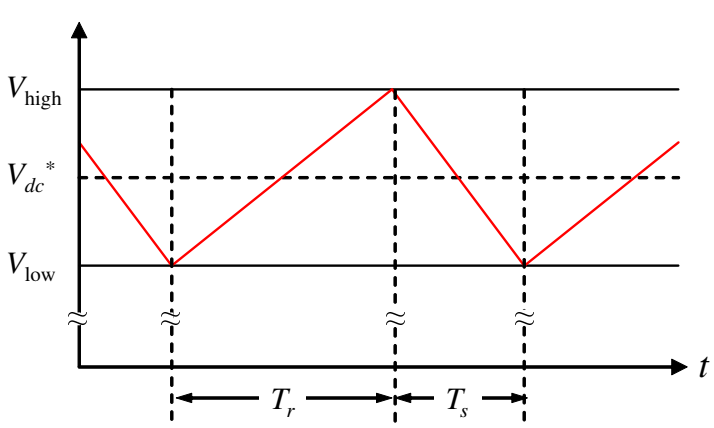

Figure 6: Waveform of the DC link voltage.

\section{Efficiency Maximization by Half Active Rectifier}

\subsection{DC link voltage control}

The HAR regulates the DC link voltage $V_{d c}$ for efficiency maximization. $V_{d c}$ control is achieved using two operation modes of HAR, which are shown in Figure 5 [12]. When the lower arm MOSFETs are off-state, HAR is operated as the rectification mode. If the MOSFETs are turned on, HAR becomes the short mode and the receiver is shorted.

Assuming the transmitting power $P$ is larger than the load power $P_{L}, V_{d c}$ is increased during the rectification mode. On the other hand, $V_{d c}$ is decreased during the short mode because $P$ is cut-off and $P_{L}$ is supplied by the DC link capacitor. Therefore, the waveform of $V_{d c}$ can be depicted in Figure 6 . If the upper bound $V_{\text {high }}$ and the lower bound $V_{\text {low }}$ are defined as follows:

$$
\begin{aligned}
& V_{\text {high }}=V_{d c}{ }^{*}+\Delta V \\
& V_{\text {low }}=V_{d c}{ }^{*}-\Delta V
\end{aligned}
$$

where $V_{d c}{ }^{*}$ is the reference value of the DC link voltage and $\Delta V$ is the hysteresis band, $V_{d c}$ is kept within the desired range.

\subsection{Efficiency maximization}

In order to maximize the transmitting efficiency, the load resistance $R_{L}$, which is expressed in Figure 2, has to satisfy eq. (4) during the rectification mode. If $V_{d c}{ }^{*}$ is given as follows:

$$
V_{d c \eta \max }=\sqrt{\frac{R_{2}}{R_{1}}} \frac{\omega_{0} L_{m}}{\sqrt{R_{1} R_{2}+\left(\omega_{0} L_{m}\right)^{2}}+\sqrt{R_{1} R_{2}}} V_{S}
$$

$R_{L}$ is equated to $R_{L \eta \max }$ and the transmitting efficiency $\eta$ can be maximized [13].

On the other hand, during the short mode, the transmitting power $P$ is drastically deceased because $R_{L}$ is minimized. As a result, losses during the short mode are assumed to be negligible to losses during the rectification mode in this paper. Therefore, $V_{d c}{ }^{*}$ is determined only by $V_{d c \eta \max }$. 


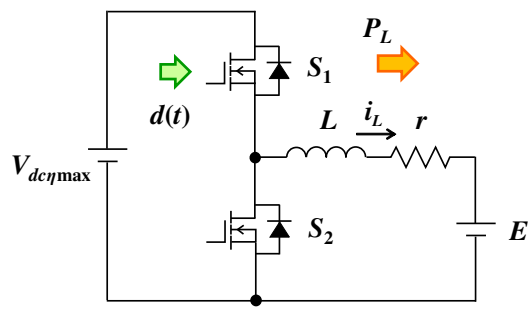

(a) Simplified DC-DC converter

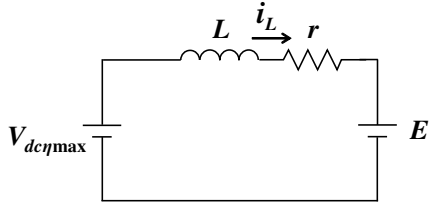

(b) $S_{1}: \mathrm{ON}, S_{2}: \mathrm{OFF}$

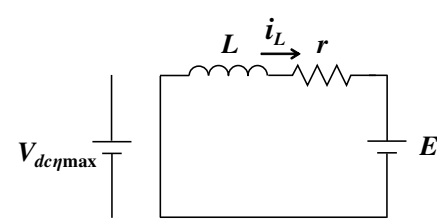

(c) $S_{1}:$ OFF, $S_{2}:$ ON

Figure 7: Circuit diagram of the DC-DC converter.

\subsection{Mutual inductance estimation}

For tracking the maximum efficiency in the DWPT system, the mutual inductance $L_{m}$ has to be estimated to obtain $V_{d c \eta \max }$ only using the vehicle-side information. From the circuit equations of the DWPT system, the estimation equation of $L_{m}$ can be given as follows [14]:

$$
\hat{L}_{m}=\frac{V_{1} \pm \sqrt{V_{1}^{2}-4 R_{1} I_{2}\left(V_{2}+R_{2} I_{2}\right)}}{2 I_{2} \omega_{0}} .
$$

Although eq. (8) has two solutions, the solution with a positive sign is used in this paper. Assuming the RMS primary voltage $V_{1}$ is constant and given to simplify ground facilities, $L_{m}$ can be estimated from the vehicle side. The RMS secondary voltage $V_{2}$ and the RMS secondary current $I_{2}$ are calculated from the DC link voltage $V_{d c}$ and the rectified DC current $I_{d c}$ using Fourier series expansions.

In order to reduce the estimation error due to the sensor noise, recursive least square (RLS) filter is applied. From eq. (8), output $y[i]$ and regressor $\varphi[i]$ are determined as follows:

$$
\begin{aligned}
y[i] & =V_{1}+\sqrt{V_{1}^{2}-4 R_{1} I_{2}[i]\left(V_{2}[i]+R_{2} I_{2}[i]\right)} \\
\varphi[i] & =2 I_{2}[i] \omega_{0} .
\end{aligned}
$$

RLS filter is used to estimate $L_{m}$ statistically by updating $\hat{L}_{m}[i], y[i]$ and $\varphi[i]$ with following equations.

$$
\begin{aligned}
\hat{L}_{m}[i] & =\hat{L}_{m}[i-1]+\frac{\varphi[i] P[i-1]}{\lambda+\varphi[i]^{2} P[i-1]} \epsilon[i] \\
\epsilon[i] & =y[i]-\varphi[i] \hat{L}_{m}[i-1] \\
P[i] & =\frac{1}{\lambda}\left\{P[i-1]-\frac{\varphi[i]^{2} P[i-1]^{2}}{\lambda+\varphi[i]^{2} P[i-1]}\right\}
\end{aligned}
$$

where $\lambda$ is forgetting factor. The initial values are given as $\hat{L}_{m}[0]=0$ and $P[0]=1$.

In order to use the effective values for the estimation, the RLS filter updates $\hat{L}_{m}[i], y[i]$ and $\varphi[i]$ only during the rectification mode of the HAR. If the HAR is operated as the short mode, $I_{d c}$ equals to 0 and the estimated value becomes incorrect. Therefore, the RLS filter has to be improved according to the operation mode of the HAR.

\section{Power Control by the DC-DC converter}

\subsection{Modeling of the DC-DC converter}

The DC-DC converter controls the load current $i_{L}$ for battery charging. Assuming the DC link voltage $V_{d c}$ is controlled to $V_{d c} \eta \max$ by the HAR, the circuit diagram of the DC-DC converter can be expressed in Figure 7 (a). $E$ is the battery voltage, $L$ is the inductance of the reactor coil and $r$ is the internal resistance of the reactor coil and the battery. In this paper, the DC-DC converter model is obtained by the state space averaging method. Assuming the DC-DC converter is operated in the continuous conduction mode, its circuit diagram in each switching modes is expressed in Figure 7 (b) and (c). 


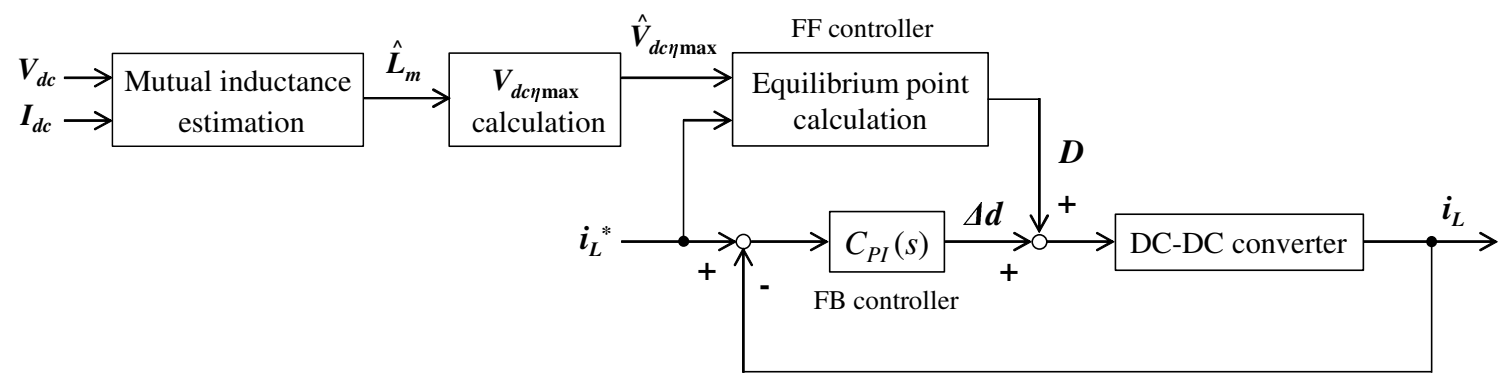

Figure 8: Block diagram of the proposed control.

From the circuit equation, the state equation of Figure 7 (b) is given as follows:

$$
\frac{d}{d t} i_{L}(t)=-\frac{r}{L} i_{L}(t)-\frac{1}{L} E+\frac{1}{L} V_{d c \eta \max } .
$$

Also, the state equation of Figure 7 (c) is described as follows:

$$
\frac{d}{d t} i_{L}(t)=-\frac{r}{L} i_{L}(t)-\frac{1}{L} E .
$$

When $d(t)$ is defined as the duty cycle of the upper arm MOSFET $S_{1}$, the state space model of the DC-DC converter is obtained as follows:

$$
\frac{d}{d t} i_{L}(t)=-\frac{r}{L} i_{L}(t)-\frac{1}{L} E+\frac{V_{d c \eta \max }}{L} d(t) .
$$

In order to apply the linear control theory to the controller design, this model is linearized around an equilibrium point. When $I_{L}$ and $D$ are defined as the equilibrium point, $i_{L}(t)$ and $d(t)$ are expressed as follows:

$$
\begin{aligned}
& i_{L}(t)=I_{L}+\Delta i_{L}(t) \\
& d(t)=D+\Delta d(t),
\end{aligned}
$$

where $\Delta i_{L}(t)$ and $\Delta d(t)$ are the microscopic fluctuations around the equilibrium point.

By substituting eq. (15) and eq. (16) in eq. (14), the linearized DC-DC converter model is given as follows:

$$
\frac{d}{d t} \Delta i_{L}(t)=-\frac{r}{L} \Delta i_{L}(t)+\frac{V_{d c \eta \max }}{L} \Delta d(t) .
$$

Therefore, the transfer function from $\Delta d(s)$ to $\Delta i_{L}(s)$ is obtained as follows:

$$
\Delta P_{i}(s)=\frac{\Delta i_{L}(s)}{\Delta d(s)}=\frac{V_{d c \eta \max }}{L s+r} .
$$

\subsection{Controller design}

Figure 8 shows the block diagram of the proposed control. The feedforward controller is the same as the equilibrium point calculation, which is given by the constraint of the DC-DC converter. From $\hat{V}_{d c \eta \max }$, which is calculated from $\hat{L}_{m}$, and the reference value of the load current $i_{L}{ }^{*}$, the equilibrium point is obtained as follows:

$$
\begin{aligned}
I_{L} & =i_{L}{ }^{*} \\
D & =\frac{E+r I_{L}}{\hat{V}_{d c \eta \max }} .
\end{aligned}
$$




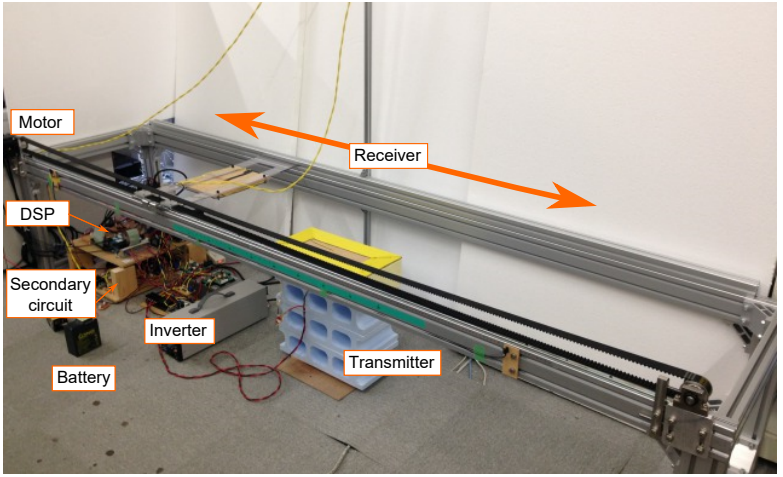

(a) Whole picture of the DWPT system.

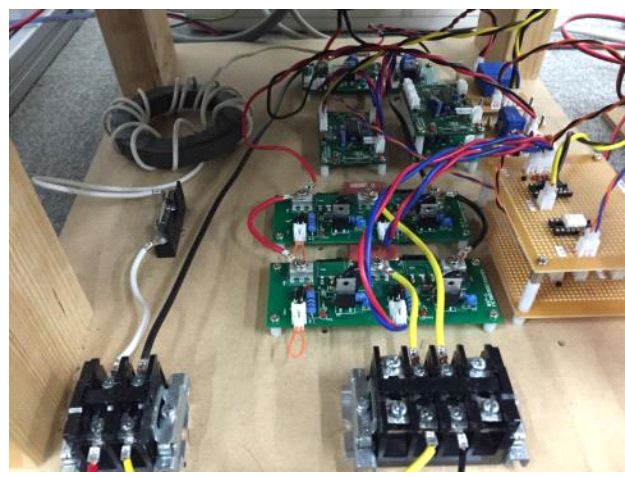

(b) Half Active Rectifier and the DC-DC converter.

Figure 9: Experimental setup.

Table 2: Simulation and experimental conditions.

\begin{tabular}{c|c||c|c}
\hline Parameter & Value & Parameter & Value \\
\hline Power source voltage $V_{S}$ & $18 \mathrm{~V}$ & Battery voltage $E$ & $6 \mathrm{~V}$ \\
Operating frequency $f_{0}$ & $100 \mathrm{kHz}$ & Reactor resistance $r$ & $0.3 \Omega$ \\
Hysteresis band $\Delta V$ & $0.5 \mathrm{~V}$ & Reactor inductance $L$ & $1000 \mu \mathrm{H}$ \\
Carrier frequency $f_{c}$ & $20 \mathrm{kHz}$ & DC link capacitance $C_{d c}$ & $2000 \mu \mathrm{F}$ \\
\hline
\end{tabular}

The feedback controller is designed by the pole placement method. As $\Delta P_{i}(s)$ is the first-order system, we apply a PI controller $C_{P I}(s)$, which is expressed as follows:

$$
C_{P I}(s)=\frac{s K_{P}+K_{I}}{s} .
$$

If closed loop poles are given by a multiple root $\omega_{c l}$, the gains are obtained as follows:

$$
\begin{aligned}
K_{P} & =\frac{2 L \omega_{c l}-r}{V_{d c \eta \max }} \\
K_{I} & =\frac{L \omega_{c l}^{2}}{V_{d c \eta \max }} .
\end{aligned}
$$

In this paper, $V_{d c \eta \max }$ and the gains are calculated by assuming the nominal value of $L_{m}$ is $30 \mu \mathrm{H}$.

\section{Simulation and Experiment}

\subsection{Experimental setup}

The experimental setup is shown in Figure 9. The system configuration is the same as Figure 4. The receiver is driven by the motor to simulate motion of the vehicle. The inverter is operated only while the receiver is above the transmitter to prevent huge power losses.

Simulation and experimental conditions are expressed in Table 2. The forgetting factor $\lambda$ of the RLS filter was set to 0.95 and the estimated mutual inductance $\hat{L}_{m}$ was updated only during the rectification mode of the HAR. The reference value of the DC link voltage $\hat{V}_{d c \eta \max }$ was calculated from $\hat{L}_{m}$ and the reference value of the load current $i_{L}{ }^{*}$ was set to $1.0 \mathrm{~A}$.

\subsection{Simulation}

In the simulations, the change in $L_{m}$ was simulated by a sine wave. Its minimum and maximum values were set to $20 \mu \mathrm{H}$ and $40 \mu \mathrm{H}$. 


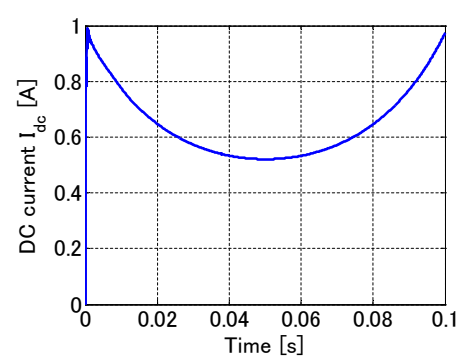

(a) DC current $I_{d c}$

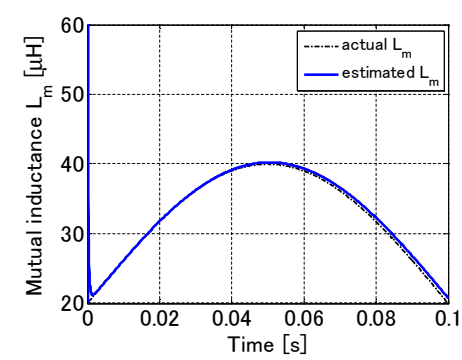

(b) Mutual inductance $L_{m}$

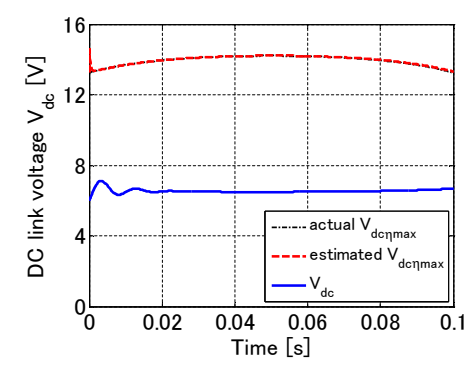

(c) DC link voltage $V_{d c}$

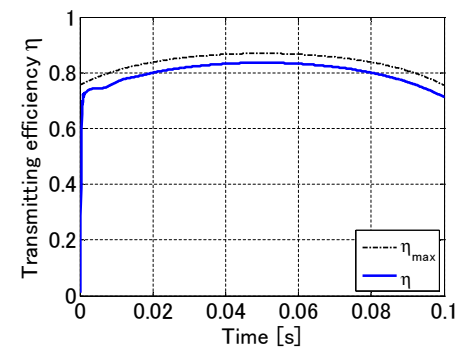

(d) Transmitting efficiency $\eta$

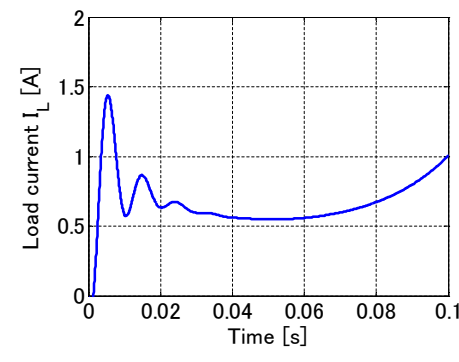

(e) Load current $i_{L}$

Figure 10: Simulation results without the proposed control.

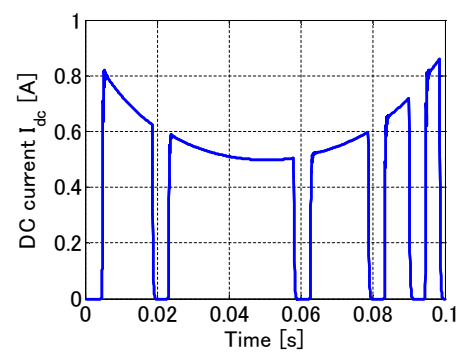

(a) DC current $I_{d c}$

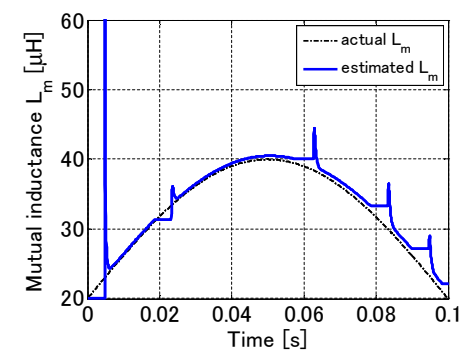

(b) Mutual inductance $L_{m}$

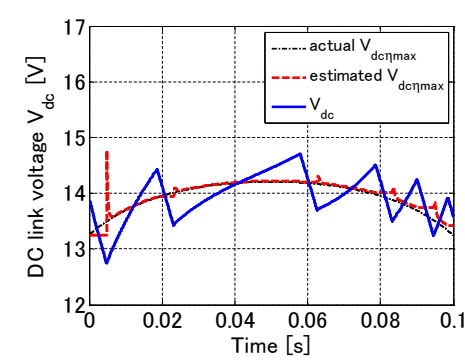

(c) DC link voltage $V_{d c}$

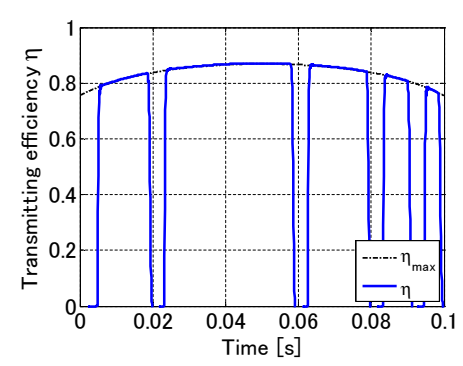

(d) Transmitting efficiency $\eta$

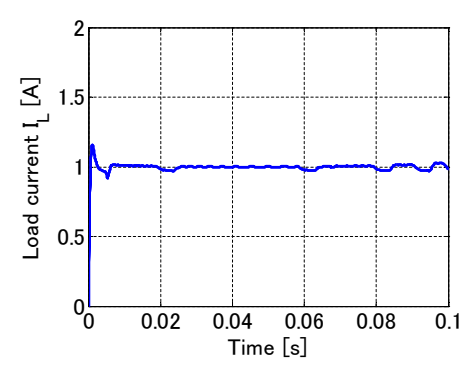

(e) Load current $i_{L}$

Figure 11: Simulation results with the proposed control.

Figure 10 shows the simulation results without the proposed control. In this simulation, the HAR was operated as only the rectification mode and the duty cycle $d$ of the DC-DC converter was equated to 0.95 . From Figure 10 (b), $\hat{L}_{m}$ is closely matched with the actual $L_{m}$. However, the transmitting efficiency $\eta$ is decreased from the maximum efficiency because the DC link voltage $V_{d c}$ cannot be regulated to $V_{d c \eta \max }$. Furthermore, the load current $i_{L}$ cannot be controlled to $i_{L}{ }^{*}$.

Figure 11 shows the simulation results with the proposed control. The closed loop poles of the proposed control were placed at $-2000 \mathrm{rad} / \mathrm{s}$. Although $L_{m}$ was estimated only during the rectification mode of the HAR, $\hat{L}_{m}$ accords with the actual $L_{m}$ as shown in Figure 11 (b). From Figure 11 (c) and (d), $V_{d c}$ is regulated around $V_{d c \eta \max }$ and $\eta$ is maximized during the rectification mode. In addition, Figure 11 (e) indicates that the load current control can be achieved. 


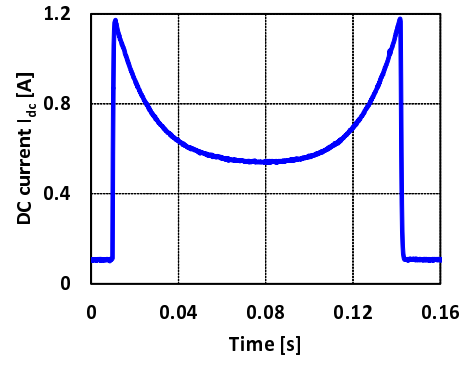

(a) DC current $I_{d c}$

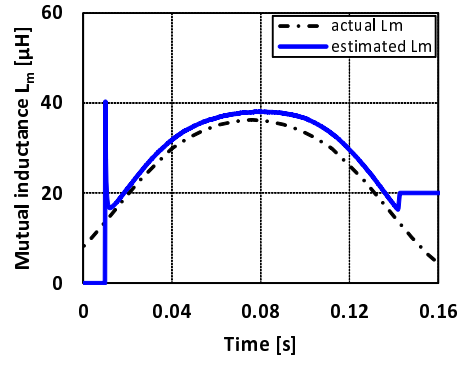

(b) Mutual inductance $L_{m}$

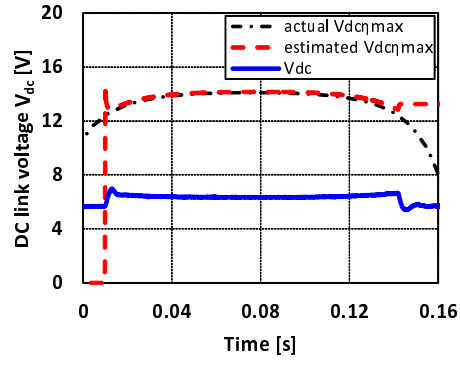

(c) DC link voltage $V_{d c}$

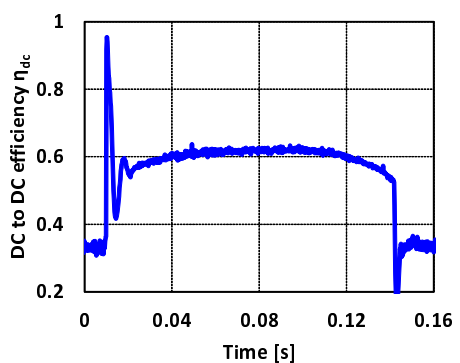

(d) DC to DC efficiency $\eta_{d c}$

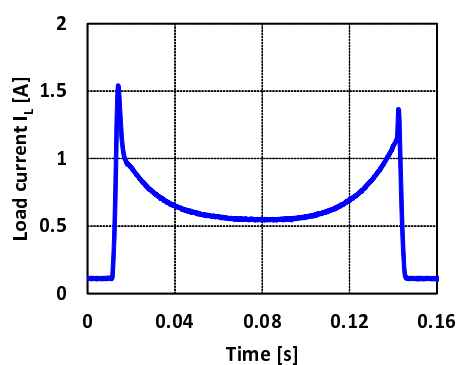

(e) Load current $i_{L}$

Figure 12: Experimental results without the proposed control.

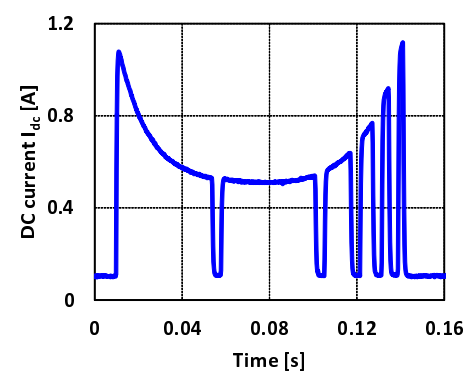

(a) DC current $I_{d c}$

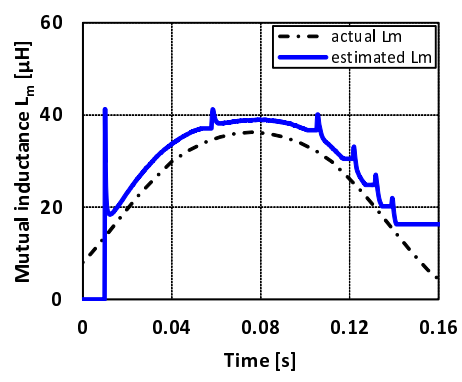

(b) Mutual inductance $L_{m}$

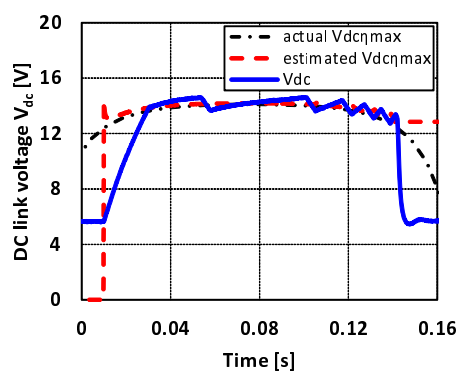

(c) DC link voltage $V_{d c}$

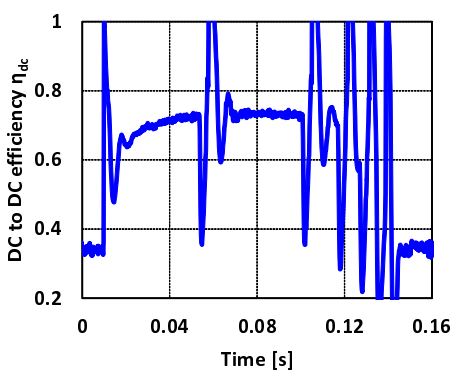

(d) DC to DC efficiency $\eta_{d c}$

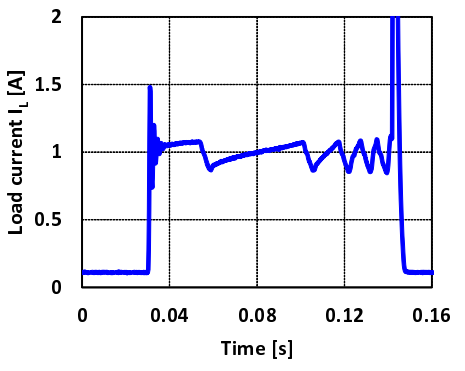

(e) Load current $i_{L}$

Figure 13: Experimental results with the proposed control.

\subsection{Experiment}

In the experiments, the receiver was driven at $10 \mathrm{~km} / \mathrm{h}$ and $\hat{L}_{m}$ was compared to the actual $L_{m}$, which was measured by an LCR meter (NF Corp., ZM2371). The DC to DC efficiency $\eta_{d c}$ includes not only the transmitting efficiency but also the converter efficiency because it was measured by the DC voltages and currents on each sides. Therefore, improvement of system efficiency is verified in the experiments. 
Figure 12 shows the experimental results without the proposed control. The HAR and the DC-DC converter were operated at the same condition as the simulation without control. From Figure 12 (b), $\hat{L}_{m}$ and the actual $L_{m}$ are closely matched. Although $\hat{L}_{m}$ has a short-time delay, $\hat{V}_{d c \eta \text { max }}$ is near by the actual $V_{d c \eta \max }$ as shown Figure 12 (c). However, the transmitting efficiency cannot be maximized because $V_{d c}$ is not regulated to $V_{d c \eta \max }$. Furthermore, Figure 12 (e) indicates that $i_{L}$ cannot be controlled unless the proposed control is applied.

In the case of with control, the DC-DC converter started power control when $V_{d c}$ reached $V_{d c \eta \max }$. The closed loop poles of the proposed control were placed at $-1000 \mathrm{rad} / \mathrm{s}$.

Figure 13 shows the experimental results with the proposed control. Although the error of $\hat{L}_{m}$ is larger than without control, $V_{d c}$ can be controlled around $V_{d c \eta \max }$ as shown in Figure 13 (c). From Figure 13 (d), $\eta_{d c}$ during the rectification mode of the HAR is increased compared to without control. In addition, Figure 13 (e) shows that $i_{L}$ can be controlled to $i_{L}{ }^{*}$. If the closed loop poles of the proposed control are optimized, it is possible to suppress the current ripple due to the fluctuation of $V_{d c}$.

\section{Conclusion}

This paper proposed a simultaneous control method of real-time power control and efficiency maximization based on improved mutual inductance estimation from the vehicle side. Its control strategy and controller design methods were presented. The effectiveness of the proposed method was verified by the simulations and the experiments.

Future works are to propose an efficiency maximization method considering losses during the short mode of HAR and to design an optimal controller for the proposed control. Furthermore, the proposed method is implemented to an actual DWPT system using an EV.

\section{Acknowledgments}

This work was partly supported by JSPS KAKENHI Grant Number 25709020 and 15H02232.

\section{References}

[1] G. A. Covic and J. T. Boys, "Modern trends in inductive power transfer for transportation application," IEEE Journal of Emerging and Selected Topics in Power Electronics, vol. 1, no.1, pp. 28-41, Mar. 2013.

[2] S. Li and C.C. Mi, "Wireless power transfer for electric vehicle applications," IEEE Journal of Emerging and Selected Topics in Power Electronics, vol. 3, no.1, pp. 4-17, Mar. 2015.

[3] J. Shin, S. Shin, Y. Kim, S. Ahn, S. Lee, G. Jung, S. Jeon, and D. Cho, "Design and implementation of shaped magnetic-resonance-based wireless power transfer system for roadway-powered moving electric vehicles," IEEE Transactions on Industrial Electronics, vol. 61, no. 3, pp. 1179-1192, Mar. 2014.

[4] J. M. Miller, O. C. Onar, C. White, S. Campbell, C. Coomer, L. Seiber, R. Sepe, and M. Chinthavali, "Demonstrating dynamic charging of an electric vehicle: the benefit of electrochemical capacitor smoothing," IEEE Power Electronics Magazine, vol. 1, no.1, pp. 12-24, Mar. 2014.

[5] K. Lee, Z. Pantic, and S. M. Lukic, "Reflexive Field Containment in Dynamic Inductive Power Transfer Systems," IEEE Transactions on Industrial Electronics, vol. 29, no. 9, pp. 4592-4602, Sep. 2014.

[6] L. Chen, G. R. Nagendra, J. T. Boys, and G. A. Covic, "Double-coupled systems for IPT roadway applications," IEEE Journal of Emerging and Selected Topics in Power Electronics, vol. 3, no.1, pp. 37-49, Mar. 2015.

[7] G. Lovison, M. Sato, T. Imura, and Y. Hori, "Secondary-side-only simultaneous power and efficiency control for two converters in wireless power transfer system," in 41st Annual Conference of the IEEE Industrial Electronics Society (IECON), 2015, pp. 4824-4829.

[8] K. Hata, T. Imura, and Y. Hori, "Dynamic wireless power transfer system for electric vehicle to simplify ground facilities - power control and efficiency maximization on the secondary side -," in Proc. 31st Annual IEEE Applied Power Electronics Conference and Exposition, 2016, pp. 1731-1736. 
[9] A. Kurs, A. Karalis, R. Moffatt, J. D. Joannopoulos, P. Fisher, and M. Soljacic, "Wireless power transfer via strongly coupled magnetic resonance," Science Express on 7 June 2007, vol. 317, no. 5834, pp. 83-86, Jun. 2007.

[10] T. Imura and Y. Hori, "Maximizing air gap and efficiency of magnetic resonant coupling for wireless power transfer using equivalent circuit and Neumann formula," IEEE Transactions on Industrial Electronics, vol. 58, no. 10, pp. 4746-4752, Oct. 2011.

[11] M. Kato, T. Imura, and Y. Hori, "New characteristics analysis considering transmission distance and load variation in wireless power transfer via magnetic resonant coupling," in IEEE 34th International Telecommunications Energy Conference (INTELEC), 2012, pp. 1-5.

[12] D. Gunji, T. Imura, and H. Fujimoto, "Basic study of transmitting power control method without signal communication for wireless in-wheel motor via magnetic resonance coupling," in Proc. The IEEE/IES International Conference on Mechatronics (ICM), 2015, pp. 313-318.

[13] M. Kato, T. Imura, and Y. Hori, "Study on maximize efficiency by secondary side control using DC-DC converter in wireless power transfer via magnetic resonant coupling," in Proc. The 27th International Electric Vehicle Symposium and Exhibition (EVS), 2013, pp. 1-5.

[14] D. Kobayashi, T. Imura, and Y. Hori, "Real-time coupling coefficient estimation and maximum efficiency control on dynamic wireless power transfer for electric vehicles," in Proc. IEEE PELS Workshop on Emerging Technologies; Wireless Power, 2015, pp. 1-6.

\section{Authors}

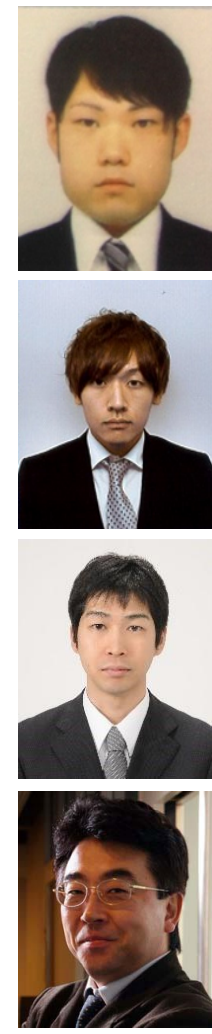

Mr. Katsuhiro Hata received his B.E. degree in electrical engineering from Ibaraki National College of Technology, Ibaraki, Japan. He received his M.S. degree in Frontier Sciences from The University of Tokyo in September 2015. He is currently working toward a Ph.D. degree at the Graduate School of Engineering with the University of Tokyo. His research interests are mainly on wireless power transfer via magnetic resonant couplings.

Mr. Daita Kobayashi received his B.S. degree in applied physics from Tokyo University of Science in 2014. He is currently working toward a M.S. degree at the Graduate School of Frontier Sciences with the University of Tokyo. His research interests are mainly on wireless power transfer via magnetic resonant couplings.

Dr. Takehiro Imura received his B.S. degree in electrical and electronics engineering from Sophia University, Tokyo, Japan. He received his M.S. degree and Ph.D. in Electronic Engineering from The University of Tokyo in March 2007 and March 2010 respectively. He is currently a Specially Appointed Associate in the Graduate School of Engineering in the same university.

Dr. Yoichi Hori received his Ph.D. in electrical engineering from The University of Tokyo, Japan, 1983, where he became a Professor in 2000. In 2008, he moved to the Department of Advanced Energy, Graduate School of Frontier Sciences. Prof. Hori was the recipient of the Best Paper Award from the IEEE Transactions on Industrial Electronics in 1993, 2001 and 2013 and of the 2000 Best Paper Award from the Institute of Electrical Engineers of Japan (IEEJ). He is the Chairman of the Motor Technology Symposium of the Japan Management Association. 